\title{
Simulation of Widebandgap Multi-Quantum Well Light Emitting Diodes
}

\author{
D. ORIATO*, ALISON B. WALKER and W. N. WANG \\ Department of Physics, University of Bath, Bath BA2 7AY, UK
}

\begin{abstract}
Charge transport has been simulated in a novel two quantum well $\mathrm{InGaN} / \mathrm{GaN}$ light emitting diode. Asymmetric tunnelling for holes and electrons has been used to enhance the quantum efficiency of the diode. A self-consistent solution of Poisson and Schrödinger equations has been used to obtain the band profile, energy levels and wave functions. Transport in the bulk nitride has been simulated by a drift diffusion model. Lattice strain and the resulting piezoelectric field effects have been shown to influence the device characteristics.
\end{abstract}

Keywords: Drift-diffusion; Schrödinger; Piezoelectric; Tunnelling; Nitrides; Heterostructure

\section{INTRODUCTION}

In the last 5 years, III-nitrides have attracted enormous attention because of their wide bandgaps. Now that material growth of sufficient quality has been achieved, they are being exploited commercially for blue and green light emitting diodes (LEDs) [1]. Though these materials have much practical importance, they are still technologically immature. As a result, their basic transport properties are not yet fully understood.

Here we present a model of a coupled quantumwell pn diode [2]. The predicted band profile, which illustrates the device structure, is shown in Figure 1. The device consists of a substrate and buffer layer (not shown) on top of which the following layers are grown (i) a $3 \mu \mathrm{m}$ thickness of GaN with n-doping of $10^{18} \mathrm{~cm}^{-3}$ (n-contact layer) (ii) a $20 \mathrm{~nm}$ thickness of $\operatorname{In}_{0.15} \mathrm{Ga}_{0.85} \mathrm{~N}$ with $\mathrm{n}$ doping of $10^{15} \mathrm{~cm}^{-3}$ (the wide well) (iii) a $2 \mathrm{~nm}$ thickness of $\mathrm{GaN}$ with $\mathrm{n}$-doping of $10^{18} \mathrm{~cm}^{-3}$ (the barrier between wide and narrow wells) (iv) a $3 \mathrm{~nm}$ thickness of $\mathrm{In}_{0.2} \mathrm{Ga}_{0.8} \mathrm{~N}$ with n-doping of $10^{15} \mathrm{~cm}^{-3}$ (the narrow well) (v) a $500 \mathrm{~nm}$ thickness of $\mathrm{GaN}$ with $\mathrm{p}$-doping of $10^{18} \mathrm{~cm}^{-3}$ (p-contact layer). Since the critical thickness of $\operatorname{In}_{x} \mathrm{Ga}_{1-x} \mathrm{~N}$ $(0<x<0.15)$ exceeds $40 \mathrm{~nm}$ [3], coherent growth of $\mathrm{InGaN}$ is assumed.

The aim of the design is to maximise light emission from the active well, to produce a narrow

*Corresponding author. Tel.: +1 22532 3174, Fax: +1 22582 6110, e-mail: pypdo@bath.ac.uk 


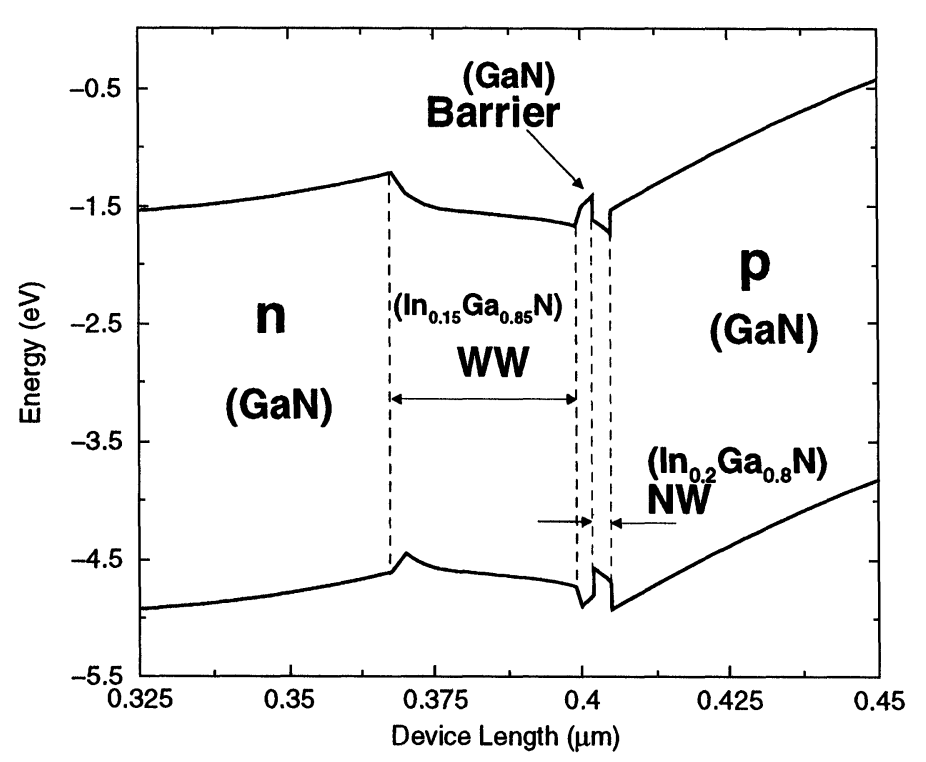

FIGURE 1 Device band profile under a bias of IV.

emission spectrum. The wide well serves to capture the electrons injected from the $n$-doped contact. Capture of the holes into the narrow well from the p-doped contact is possible because of the large hole effective mass. By adjusting the alloy composition in the wide and narrow wells, and choosing the correct width for the narrow well, it is possible to match the position of the energy of the lowest electron sub-band in the wide well with the energy of the lowest sub-band in the narrow well, thereby creating a high tunneling probability for the electrons from the wide to the narrow well. At the same time, the highest energy hole sub-band in the narrow well should stay above the bottom of the valence band in the wide well, thus hole penetration into the wide well can only occur with thermal activation. Because of the heavy mass of holes, the width of the barrier between the wells is sufficient to prevent significant tunnelling of thermally activated holes from the narrow to the wide well.

While the idea is relevant to other materials, this structure is best exploited using nitrides. The reason is that the lattice parameter in the narrow well is close to that of the highly tensile strained thin $\mathrm{GaN}$ barrier, and the lower In concentration wide well has a lower lattice mismatch than the narrow well with the $\mathrm{n}-\mathrm{GaN}$ substrate. The wide well also stops threading dislocations arising from high stress at the $\mathrm{n}-\mathrm{GaN} / \mathrm{InGaN}$ interface.

In this paper we have linked a drift diffusion model describing macroscopic conservation of the charge, with a Schrödinger solver in the quantum wells. The models are linked by a net capture determined by thermionic emission. Because of the large mismatch between $\mathrm{GaN}$ and $\mathrm{InN}$, the effects of strain, even with low In compositions, are large [4]. Thus we have allowed for the strain-induced piezoelectric field [5] on the device performance.

\section{METHOD}

Our simulator consists of a steady-state onedimensional drift diffusion model assuming nondegenerate statistics [6] in which the continuity equations are coupled to the Poisson equation, in turn coupled to a Schrödinger equation to give the bound-state energies, potential profile and carrier densities within the coupled quantum wells. Materials parameters are taken from [1] and [13] with bowing parameters from [14]. 
In the continuity equation, carrier capture is represented by the net capture and escape current, which for thermionic emission is given by [7]

$$
\begin{aligned}
J_{Q W}=\frac{q k_{B}^{2} T^{2}}{2 \pi^{2} \hbar^{3}}[ & \exp \left(\frac{E_{F, B}-V_{b}+\psi}{k_{B} T}\right) \\
& \left.-\exp \left(\frac{E_{F, Q W}-V_{b}+\psi}{k_{B} T}\right)\right]
\end{aligned}
$$

Here $E_{F, B}$ and $E_{F, Q W}$ are the quasi Fermi levels of the barrier and the quantum well, $V_{b}$ is the quantum well depth and $\psi$ is the electrostatic potential. This expression guarantees zero capture current for thermal equilibrium. The region where carriers are captured into the coupled quantum wells is given by the full-width at half maximum of the probability density as calculated by the Schrödinger equation. The tunnelling current is given by [8]. We have assumed Shockley-ReadHall recombination with a lifetime of approximately $7 \mathrm{~ns}$ for holes and $0.1 \mathrm{~ns}$ for electrons [9] and a reduced lifetime in the quantum wells to allow for light emission [1].

The continuity and Poisson equations comprise a set of coupled, non-linear partial differential equations, which are solved with a numerical finite difference scheme. In each iteration, the energy band profiles and the electron and hole quasi-Fermi levels are updated by solving the Poisson equation and the electron and hole continuity equations self-consistently. Non-uniform meshes and ohmic contact boundaries were assumed. To solve the Schrödinger equation we used a transfer matrix technique $[8,10]$ in which the full-width half-maximum of a resonance in the modulus squared of the transmission coefficient was fitted to a Lorentzian for bound states to obtain the energy and lifetime of the state [11]. The oscillator strength is proportional to the square of the overlap integral [12].

\section{RESULTS}

The conduction and valence bands shown in Figure 1 increase in energy going away from the $\mathrm{n}$-contact due to the builtin voltage from the $\mathrm{n}$ and p-doped GaN contact layers, which is reduced by an external forward bias. Additional band bending occurs at the heterojunction interfaces due to bound surface charge from the polarisation induced by the piezoelectric effect.

We looked for well specifications that would achieve resonant tunnelling, viz $E_{1 n w w}=E_{1 n n w}$ where $E_{1 n w w}, E_{1 n n w}$ are respectively the energies of the lowest electron sub-bands in the wide and narrow wells. Resonance between higher subbands in the wide well and the lowest sub-band in the narrow well is not desirable as the former states are less populated, reducing the tunnelling current. We found that the ratio between the length of the wide well $l_{w w}$ to that of the narrow well $l_{n w}$ has to be less than 10 for resonant tunneling at zero bias. This is because as $l_{w w}$ increases, $E_{1 n w w}$ approaches the bottom of the conduction band and this is usually less than $E_{1 n n w}$. This limit can be overcome if the In composition in the wide well is reduced. As the bias voltage is increased, so does the likelihood of resonance tunnelling between the lower subbands due to flattening of the band profiles.

Figure 2 compares the band profiles for two piezoelectric polarizations. It shows that the piezoelectric field has a crucial influence on the energy level position within the wells. Our values for the piezoelectric polarization are considerably lower than the value of $1.1 \times 10^{-6} \mathrm{C} / \mathrm{cm}^{2}$ for $\mathrm{In}_{0.2} \mathrm{Ga}_{0.8} \mathrm{~N}$ on $\mathrm{GaN}$ [5] since the wide well width is close to the relaxation length and dislocations start forming, causing a reduction in the effective strain and in the polarization. At the larger polarization the wide well assumes a triangular shape, due to the band bending at the interface with the n-contact layer from the surface charge due to the piezoelectric field. While the Quantum Confined Stark Effect (QCSE) reduces $E_{1 n w w}$ the tilting of the bands from the surface charge has a much bigger effect and the net result is to raise the wide well, and so $E_{1 n w w}$ above the narrow well. Resonance tunnelling can thus be achieved as shown in Figure 2. An unwanted consequence of 

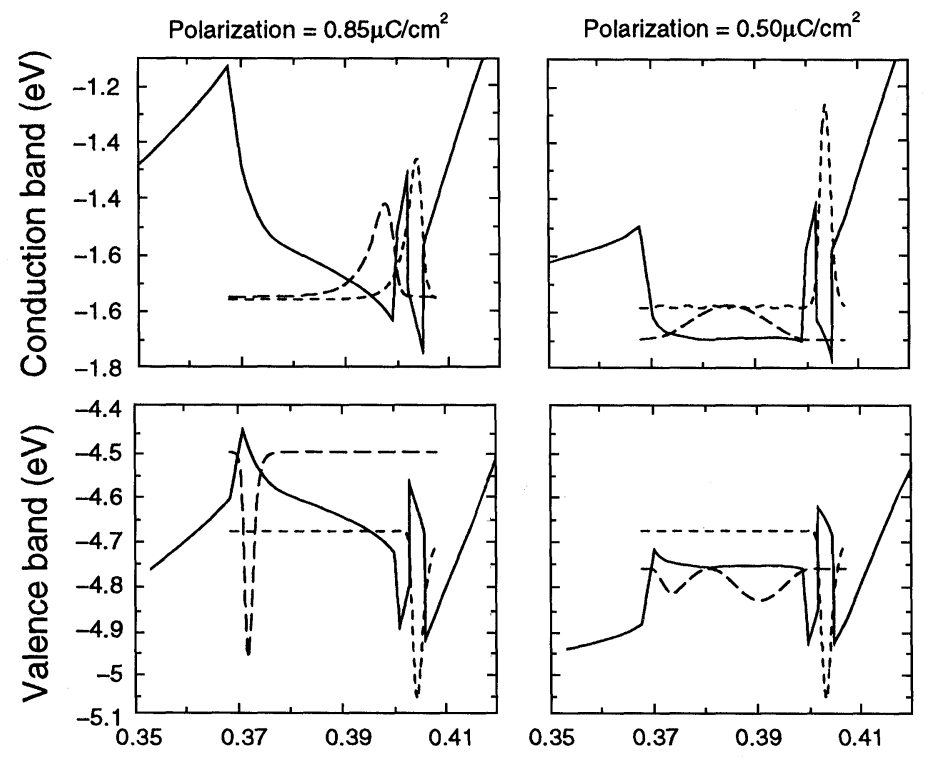

FIGURE 2 Conduction and valence band for two different values of piezoelectric field at a bias of $1 \mathrm{~V}$.

the piezoelectric field is that $E_{1 h w w}>E_{1 h n w}$, where $E_{1 h n w}$ is the energy of the highest hole subband in the wide well. Hence, hole tunnelling may occur from the narrow to the wide well. Fortunately, the valence band at the bottom of the wide well has a negative slope and the hole wavefunctions are localized far from the barrier and the narrow well, so tunnelling is negligible.

Figure 3 shows how $E_{g n w}$ depends on $l_{n w}$ for two In compositions, where we have compared $E_{g n w}$ for strained and relaxed structures to demonstrate the effect of the piezoelectric field. The electric field



FIGURE 3 Effective bandgap in the narrow well as function of the well width. Results for a strained well are compared with those of a relaxed well for indium compositions of $10 \%$ and $20 \%$. 
across the quantum well, which is the sum of the fields due to spontaneous and piezoelectric polarization and the pn junction field, causes the redshift of the ground state resonance energy $\left(E_{1 n n w}\right.$, $\left.E_{1 h n w}\right)$, a direct consequence of the QCSE discussed above. This effect can be seen in Figure 3, for the lower In composition (hence a lower piezoelectric field).

As we increase the In composition in the unstrained quantum well $E_{g n w}$ approximates the value determined from the top of the valence band and the bottom of conduction band (the real bandgap), whereas in the strained well, the reduction in $E_{g n w}$ increases rapidly with well width. This result has been explained in [15] as due to the fact that the potential drop, for high piezoelectric field and well width, becomes comparable with the conduction and valence band offsets. Hence the hole and electron confined levels drop in the triangular potential wells and $E_{g n w}$ is further reduced. The results of Figure 3 agree with an effective band energy estimate in [15] from photoluminescence. Our calculations show that the overlap of wave functions in the triangular potential wells is greatly reduced by the electric field from electrostatic attraction between electrons and holes, which opposes the piezoelectric field.

\section{CONCLUSIONS}

We have modelled a novel light emitting diode structure based on two coupled quantum wells that relies in resonance tunneling of electrons between the wide and narrow quantum wells. Our results show the importance of the piezoelectric field for the possibility of resonant tunnelling between the quantum wells. We have made predictions for the effective band gap in the narrow well when emission is introduced, which agree with experimental data [15].

\section{References}

[1] Jain, S. C. (2000). Applied Physics Reviews, 87, 965-1006.

[2] Wang, W. N. (1999). unpublished.

[3] Akasaki, I. and Amano, H. (1997). Jpn. J. Appl. Phys., 36, 5393.

[4] Nardelli, M. B., Rapcewicz, K. and Bernholc, J. (1997). Appl. Phys. Lett., 71, 3135-7.

[5] Bernardini, F., Fiorentini, V. and Vanderbilt, D. (1997). Phys. Rev. B., 56, 10024-7.

[6] Sze, S. M. (1981). Physics of Semiconductor Devices, New York: John Wiley \& Sons.

[7] Schneider, H. and Klitzing, K. (1988). Phys. Rev. B., 38, $6160-5$.

[8] Davies, J. H., The Physics of Low-Dimensional Semiconductors, Cambridge: Cambridge University Press, 1998.

[9] Bandic, Z. Z., Bridger, P. M., Piquette, E. C. and McGill, T. C. (2000). Solid-state Electronics, 44, 221-8.

[10] Sugg, A. R. and Leburton, J. P. C. (1991). IEEE J. Quantum Electron., 27, 224-231.

[11] Ghatak, A. K., Thyagarajan, K. and Shenoy, M. R. (1988). IEEE J. Quantum Electron., 24, 1524-30.

[12] Miller, D. A. B., Wiener, J. S. and Chemla, D. S. (1986). IEEE J. Quantum Electron., 22, 1816-30.

[13] Yeo, Y. C., Chong, T. C., Li, M. F. and Fan, W. J. (1988). J. Appl. Phys., 84, 1813-9.

[14] McCluskey, M. D., Van de Walle, C., Master, C., Romano, L. and Johnson, N. M. (1998). Appl. Phys. Lett., 72, 2725-6.

[15] Chichibu, S. F., Sota, T., Wada, K., DenBaars, S. and Nakamura, S. (1999). MRS Internet J. Nitride Semicond Res., 4S1, G2.7. 

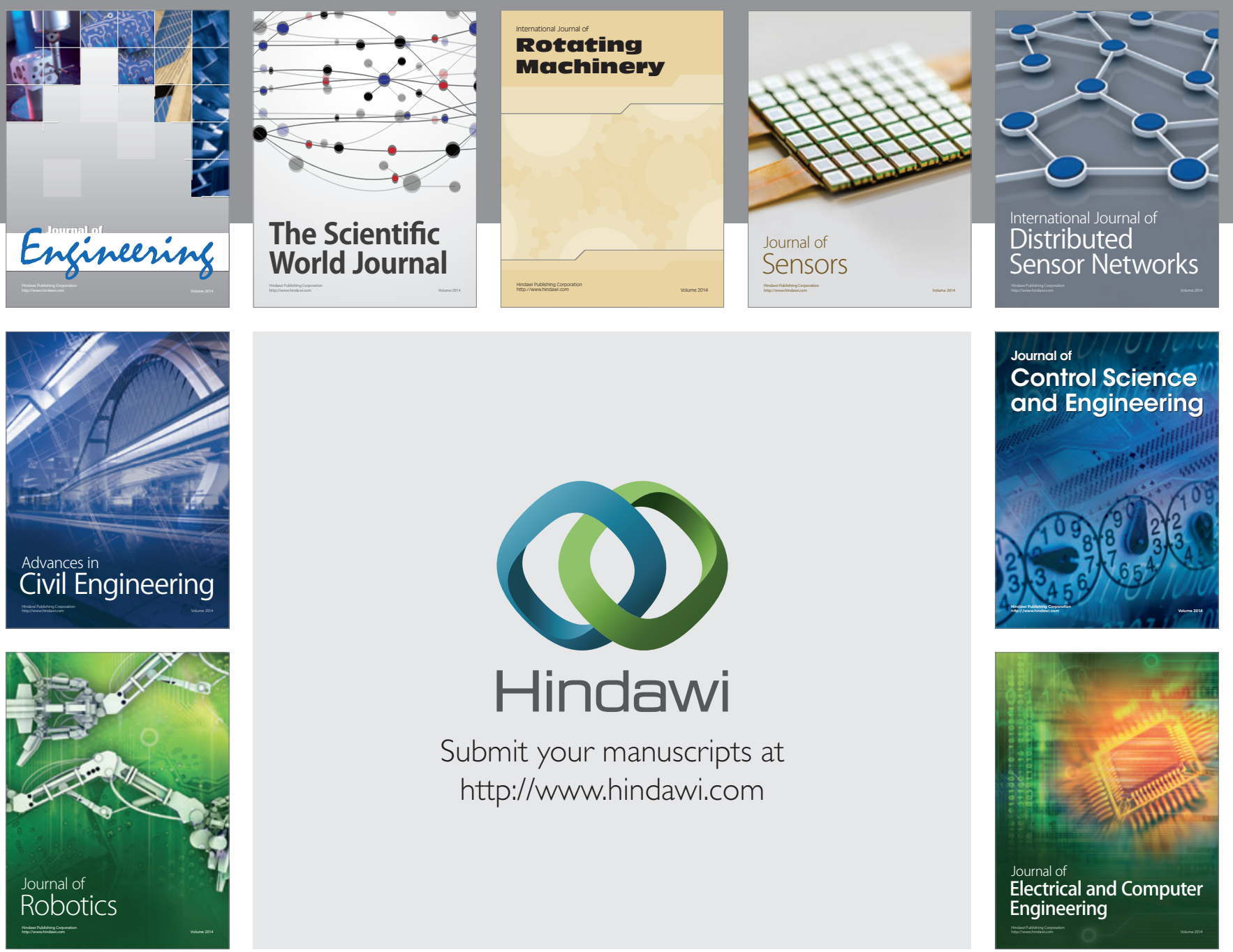

Submit your manuscripts at

http://www.hindawi.com
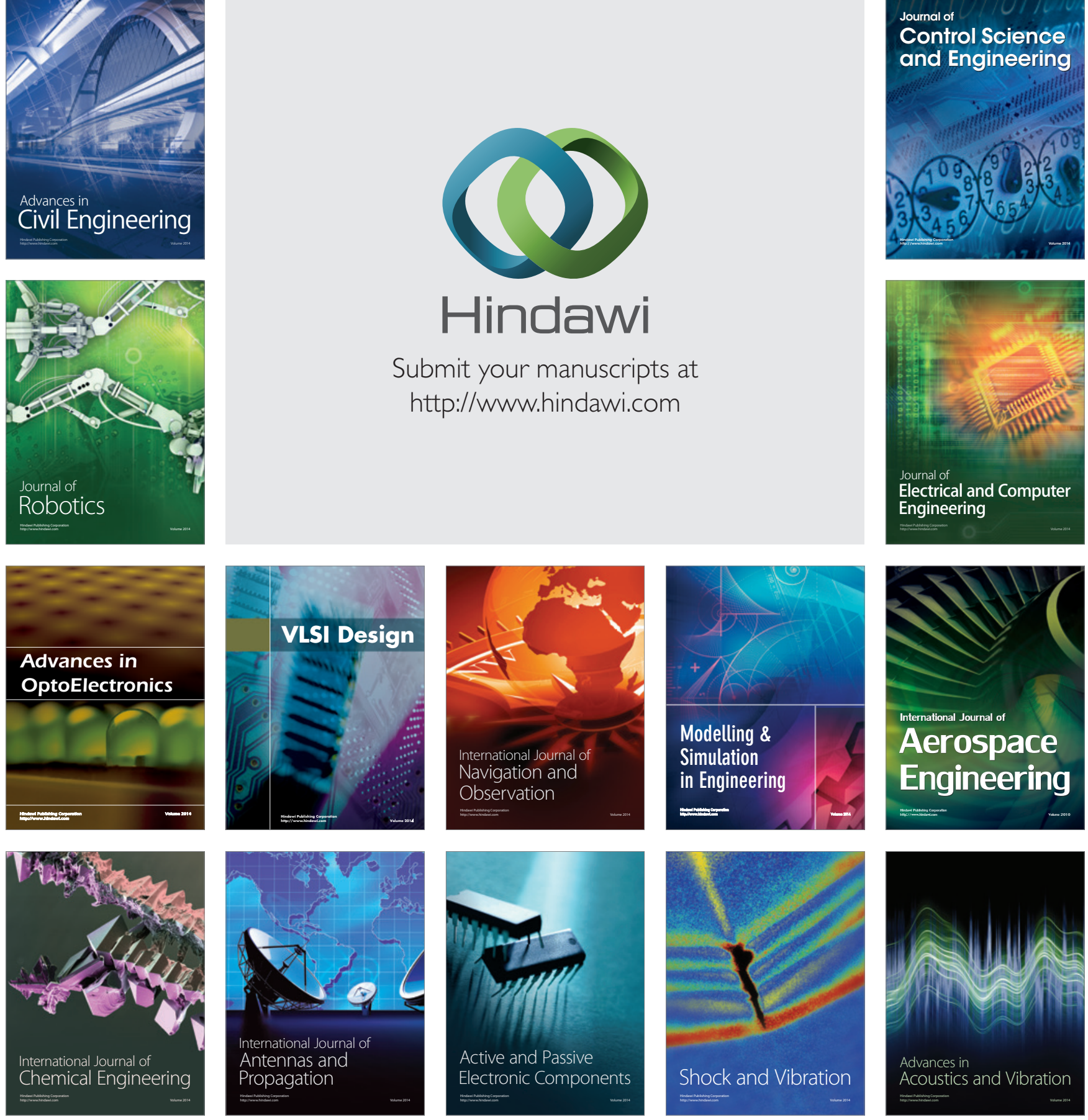